\title{
TRAVERSAL TIME THROUGH DOUBLE-BARRIER RESONANT TUNNELING STRUCTURE
}

\author{
T. FIGIELSKI \\ Institute of Physics, Polish Academy of Sciences \\ Al. Lotników 32/46, 02-668 Warszawa, Poland
}

\begin{abstract}
There are certain confusing statements in the literature concerning a physical time that is responsible for a high-frequency limit in operation of double-barrier resonant tunneling devices. Here, it is shown that an electron traversal time, introduced earlier by ours, exhibits no singularity on resonance and might be a good candidate for the quantity in question.
\end{abstract}

PACS numbers: $73.40 . \mathrm{Gk}, 85.30 . \mathrm{Mn}$

Resonant tunneling in artificially grown double-barrier resonant tunneling structures (DBTRS) has drawn much attention in recent years because of a promising possibility of its applications in high-frequency devices. It has been generally believed that tunneling is the fastest mechanism of electron transport in heterostructures. Indeed, Sollner et al. [1, 2] showed that AlGaAs-based DBRTS's grown by molecular beam epitaxy could oscillate at a frequency of $600 \mathrm{GHz}$ and detect far infrared radiation at a frequency of $2.5 \mathrm{THIz}$. These results gave rise to many debates as they seemed to be in conflict with the originally assumed Fabry-Perot (coherent) mechanism for resonant tunneling [3].

Indeed, a considerable storage time is needed to built up a steady-state probability amplitude in the quantum well which is a necessary condition for the resonance. Equivalently, this is the time spent by an electron in the quantum well where it bounces back and forth between the two barriers. It has been usually assumed that the storage time gives the fundamental speed limit in the DBRTS devices. Its value depends essentially on parameters of the structure but usually falls within the picosecond range. Luryi [4] estimated it for some investigated structure to be $40 \mathrm{ps}$, which is about three orders of magnitude too long to give an account for the extreme frequencies reported in the literature.

Therefore, Luryi has proposed an alternative explanation for the negative differential resistivity (NDR) in DBRTS, according to which an electron sequentially tunnels into the well and escapes by tunneling through the second barrier. In this case the origin of NDR would be conceptually similar to that in the Esaki diode.

It was next demonstrated by Weil and Vinter [5] that the two proposed mechanisms lead, in fact, to the same predictions for the dc current. These authors 
calculated also the storage time in the case of sequential tunneling for the same structure as considered by Luryi and obtained a value $0.4 \mathrm{ps}$, which is two orders of magnitude shorter than that given by Luryi. This difference is astonishing, since it becomes clear after some thought that the storage times for both coherent and sequential tunneling are physically identical and are determined by a leaking rate of an electron out of the quantum well. Therefore, in this respect the sequential tunneling is not a bit better from the coherent one, and the apparent difference in the storage times must follow solely from different ways of their calculations in Refs. [4] and [5].

According to our present opinion, it is not the storage time that limits the high-frequency operation of DBRTS device, at least in the low-signal limit. We considered an electron traversal time across a heterostructure with an arbitrary potential profile and proposed to describe it in a stationary case by the following expression [6]:

$$
\tau=\frac{Q}{J}
$$

where $Q=\int_{0}^{L}|\psi|^{2} \mathrm{~d} x$ is the squared modulus of a stationary wave function $\psi$ relevant to the corresponding scattering problem, integrated over the structure width, $L$, and $J$ is the probability current.

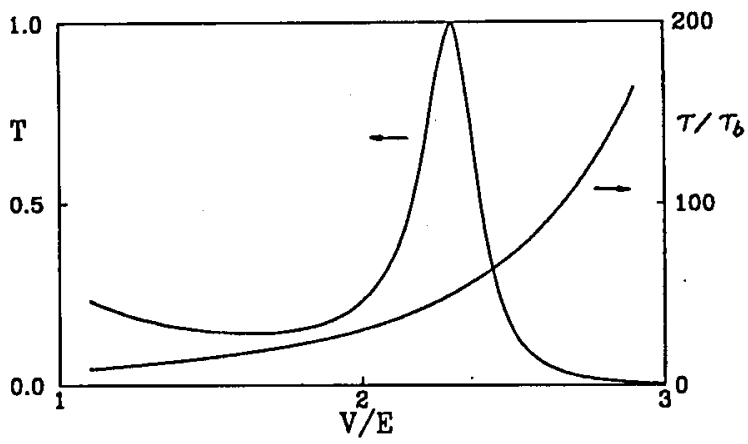

Fig. 1. Transmission coefficient, $T$, and relative traversal time, $\tau / \tau_{\mathrm{b}}$, for a symmetric DBRTS versus the ratio of the potential barrier height to the energy of incident electron, $V / E$.

This expression was originally used to examine the electron tunneling time through a single rectangular barrier. Here, this expression is applied to the case of DBRTS with the aim of examining the behavior of traversal time across the structure under condition of resonance. For this purpose the traversal time was calculated by Eq. (1) for unbiased symmetric DBRTS with the same width, $w$, of the barriers as of the well. A coherent tunneling was assumed and an usual procedure of matching the wave function and their derivatives at the boundaries of potential discontinuity in the structure was applied.

The transmission coefficient of the whole structure and the traversal time across it are shown in Fig. 1 as a function of the parameter $V / E$ (the ratio of 
the barrier height $V$ to the energy of an incident electron $E=\hbar^{2} k^{2} / 2 m$ ) for $k w=1.695$. The traversal time is expressed in units $\tau_{\mathrm{b}}$ equal to a time of ballistic motion of the incident electron across a distance corresponding to the structure width. It is seen that a resonant enhancement of the transmission coefficient through DBRTS reaches a value of unity at $V / E=2.3$.

The principal result of this investigation is that the traversal time of an electron is a monotonic function of $V / E$ within the examined range, showing no singularity on resonance. This time can explain the frequency limit for DBRTS-device operation consistently with the reported results.

In conclusion, it has been demonstrated that the electron traversal time, given by Eq. (1), behaves regularly as the structure passes through the resonance. It is proposed that this traversal time, and not the storage time, determines a high-frequency cut-off of DBRTS-device operation in the low-signal limit.

This work is supported by a grant No. 2.0461.91.01 from the Committee for Scientific Research.

\section{References}

[1] T.C.L.G. Sollner, W.D. Goodhue, P.E. Tannewald, C.D. Parker, D.D. Peck, Appl. Phys. Lett. 43, 588 (1983).

[2] E.R. Brown, T.C.L.G. Sollner, C.D. Parker, W.D. Goodhue, C.L. Chen, Appl. Phys. Lett. 55, 1777 (1989).

[3] B. Ricco, M.Ya. Azbel; Phys. Rev. B 29, 1970 (1984).

[4] S. Luryi, Appl. Phys. Lelt. 47, 490 (1985).

[5] T. Weil, B. Vinter, Appl. Phys. Lett. 50, 1281 (1987).

[6] T. Figielski, submitted to publication. 